\title{
Research on Dynamic Balance Adjustment Method of Single Braced Frame Gyroscope Rotor
}

\author{
Xukui Hou, Ende Wang, Hui Cao, Yalong Zhu, and Kai Qi
}

Key Laboratory of Optical Electrical Image Processing, Shenyang Institute of Automation, Chinese Academy of Sciences, 114, Nanta Street, Shenhe District, Shenyang, Liaoning, China

\begin{abstract}
The uneven mass distribution of gyro rotors results in vibration, rotation and drift of gyro rotors, which seriously affect the performance index and life of gyro rotors. However, because there is no rigid connection between the rotary shaft and the shell of the gyro rotor, the dynamic balancing machine can only balance the vibration component of the single braced frame gyroscope rotor, and can't measure the gyro rotor's rotation component. By analyzing the influence of uneven rotor mass distribution on the gyro rotor performance, a method of eliminating two rotational degrees of freedom of the gimbal in gyro rotor by mandrel is proposed, which makes the dynamic balancing machine directly measure the vibration component and the moving component of the gyroscope rotor, and simplifies the dynamic balancing debugging process.
\end{abstract}

\section{Introduction}

In practice, due to the uneven distribution of material quality, machining dimension error, assembling gap and other reasons, it is impossible to ensure that the center of gravity of the gyroscope rotor coincides completely with the rotation center. Because of the deviation between the center of gravity of the rotor and the center of rotation, gyroscope rotor in the work will produce unbalanced force and unbalanced moment according to D'Alembert Principle[1]. They will exert additional load on the gyro rotor bearing, unbalanced force will follow the gyro rotor rotation and produce a rotor with the same frequency of mechanical vibration, will reduce the reliability of connection, speed up the wear of bearings, reduce its service life. The unbalanced moment will produce a forced nutation on the gyro rotor and affect the imaging quality of the optical system. Therefore, it is necessary to reduce the unbalance force and moment of the gyroscope rotor to a more reasonable value and ensure the system to work properly.

At present, the balance method of the coordinator gyro rotor is mainly to adjust the unbalance force of the rotor through the dynamic balancing machine[2], and to adjust the unbalance moment of the rotor depending on the optical system and the detector imaging results. By analyzing the unbalance force and unbalance moment of the rotor, a method of improving the balancing device is proposed to simplify the balancing process of the gyro rotor.

\section{Rotor unbalance analysis}

\subsection{Decomposition and synthesis of force}

Because the gyro rotor is a single braced frame type 3 -DOF rotor, the deviation of the center of gravity and rotation of the rotor has three influences on the gyro rotor. The first is the axial deviation of the center of gravity and rotation of the rotor exerts a fixed moment on the rotor, which leads to the drift of the gyro rotor. The second is the radial deviation of the center of gravity and rotation of the rotor leads to a centrifugal force when the gyro rotor rotates, which is the same as the rotating frequency of the rotor and causes the gyroscope rotor to vibrate. The last one impact, because the rotor's diameter-width ratio is $d / b<5$, its mass cannot be regarded as distributing in the same plane, because the centrifugal force produced by each eccentric quality is not in the same rotary plane, and the inertia moment is formed, so that the gyro rotor produces a round motion. The structure diagram of gyro rotor is shown in figure 1.

The stress state of the gyro rotor is shown in figure 2, figure 3, F and $M$ are the unbalance force and unbalance moment of the gyro rotor, and the $\theta$ is the angle between the unbalance force $\mathrm{F}$ and the unbalanced moment $\mathrm{M} ; F_{F A}$ and $F_{F B}$ are the force of the unbalance force $\mathrm{F}$ on the front and back correction plane, that is the vibration component of the gyroscope rotor; $F_{M A}$ and $F_{M B}$ are the force of the unbalanced moment $\mathrm{M}$ on the front and back correction plane, that is the rotating component of the gyroscope rotor; $F_{A}$ is the resultant force of the $F_{F A}$ and $F_{M A}$, that is the unbalance on the front correction surface; $F_{B}$ is the combination of $F_{F B}$ and $F_{M B}$, that is the unbalance on the posterior correction surface; $\alpha$ is the angle between $F_{A}$ and $F_{B}$. 


$$
\begin{gathered}
F_{A}=\frac{\sqrt{F^{2} l_{2}^{2}+M^{2}-2 F l_{2} M \sin \theta}}{l_{1}+l_{2}} \\
F_{B}=\frac{\sqrt{F^{2} l_{1}^{2}+M^{2}+2 F l_{1} M \sin \theta}}{l_{1}+l_{2}}
\end{gathered}
$$

The effect of unbalance force and unbalance moment on the front and rear of the gyro rotor can be calculated by Equation 1 and 2. But in practice, $F_{A}$ and $F_{B}$ can be measured by dynamic balancing machine[3], and the unbalance force and unbalance moment of the gyroscope rotor can be calculated by Equation 3 and 4 .

$$
\begin{gathered}
F=\sqrt{F_{A}^{2}+F_{B}^{2}+2 F_{A} F_{B} \cos \alpha} \\
M=\sqrt{F_{A}{ }^{2} l_{1}^{2}+F_{B}^{2} l_{2}^{2}-2 M_{A} M_{B} \cos \alpha}
\end{gathered}
$$

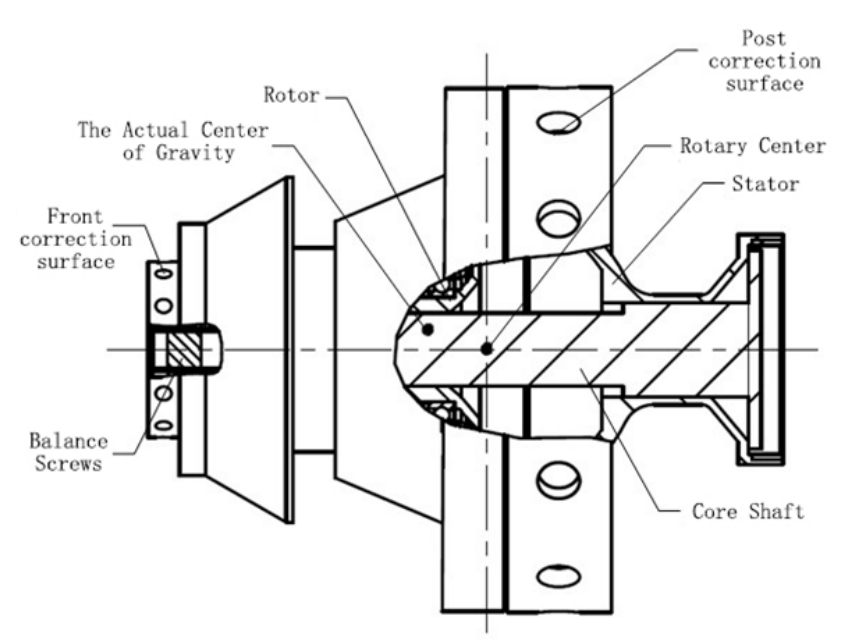

Fig. 1. Structure diagram of gyro rotor.

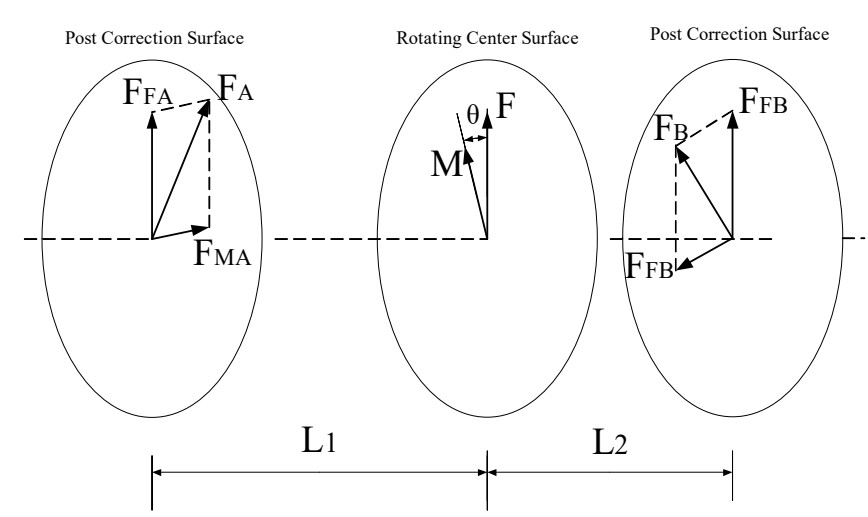

Fig. 2. The stress diagram of gyro rotor.

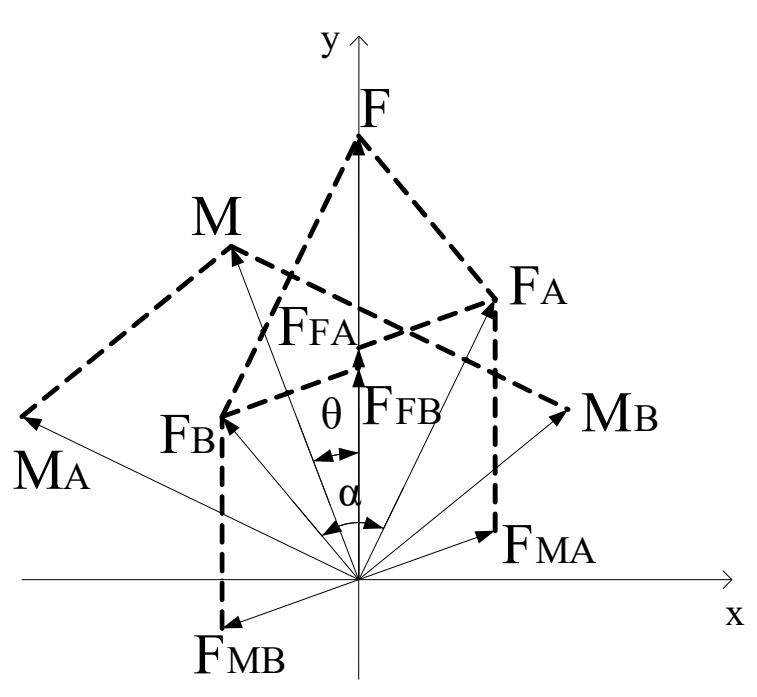

Fig. 3. The decomposition and synthesis diagram of the force and moment of gyro rotor.

\subsection{The effect of unbalanced moment $M$ on gyro rotor}

The so-called rotation is the force nutation of the gyro rotor under the action of a gyro rotor under the unbalanced moment $\mathrm{M}$, which is in a direction relative to the rotor. Because the forced nutation angle is small the higher order differential term of $\alpha$ and $\beta$ is ignored, the motion equation of the gyroscope rotor can be simplified as follows:

$$
\left.\begin{array}{l}
J_{x} \ddot{\alpha}+J_{z} \omega \dot{\beta}=M \sin \omega t \\
J_{y} \ddot{\beta}-J_{z} \omega \dot{\alpha}=-M \cos \omega t
\end{array}\right\}
$$

In the Equation: $\alpha, \beta$ are the gyro rotating shaft around the gyro inner ring axis and the rotation of the outer ring axis, $J_{x}, J_{y}, J_{z}$ are the gyroscope rotor relative to the gyro inner ring axis, outer ring axis, gyro rotating inertia, in order to simplify the calculation we set $J_{x}=J_{y} ; \omega$ is gyro rotation angular velocity.

To solve the Equation 5, you can get:

$$
\left.\begin{array}{l}
\alpha=\frac{M}{\omega^{2}\left(J_{z}-J_{x}\right)} \cdot \sin \omega t \\
\beta=-\frac{M}{\omega^{2}\left(J_{z}-J_{x}\right)} \cdot \cos \omega t
\end{array}\right\}
$$

By Equation 6 you can draw:

$$
\alpha^{2}+\beta^{2}=\left[\frac{M}{\omega^{2}\left(J_{z}-J_{x}\right)}\right]^{2}
$$

When the gyro rotor is subjected to an unbalanced moment $\mathrm{M}$, the motion trajectory of the gyro rotating shaft is a conical surface, the size of the cone angle depends on the unbalance moment $\mathrm{M}$ and the rotational angular velocity $\omega$ of the gyroscope rotor. The larger the 
angular velocity $\omega$ and the smaller the unbalance moment $\mathrm{M}$, the smaller the forced nutation angle of the gyro rotor.

\section{Adjustment method and result of gyro rotor dynamic balance}

Because the rotor is a single braced frame type 3-DOF gyroscope, the existence of the two rotational degrees of freedom of the gimbal makes the unbalance moment of the rotor unable to be transferred to the shell, and only the single balance method can be used to balance the vibration[4]. However, by adding a mandrel, the gyro stator shaft and the rotor shaft are fixed together to eliminate the two rotational degrees of freedom of the universal joint, so that the rotor becomes a double support rigid rotor, and the two-sided balance method can be used to eliminate the rotor vibration and rotation.

For unbalanced rotor, there is a phase difference angle between the unbalance centrifugal force and the amplitude caused by unbalanced weight, that is, the amplitude lags behind the unbalance force. When the rotational speed is constant, the angle is a fixed value. Moreover, when the dynamic balancing machine is calibrating, an angle error is also introduced. The combination of these two angles will make the unbalance phase of the dynamic balancing machine deviate and affect the adjustment of the rotor unbalance. The deviation can be corrected by calculating the variation of unbalance before and after correction. Suppose the unbalance amplitude and phase of the rotor correction surface measured by the dynamic balancing machine are $A_{1}, \phi_{1}$, the unbalance of the rotor is measured after adding a balance screw with mass $m$ reverse $\phi_{1}$, and the amplitude and phase of the post correction surface unbalance are $A_{2}$ and $\phi_{2}$, as shown in figure 4.

$$
\begin{aligned}
A_{3} & =\sqrt{A_{1}^{2}+A_{2}^{2}-2 A_{1} A_{2} \cos \left(\varphi_{2}-\varphi_{1}\right)} \\
\alpha & =\cos ^{-1}\left(A_{3}^{2}+A_{2}^{2}-A_{1}^{2} / 2 A_{1} A_{3}\right)
\end{aligned}
$$

The measurement error $\alpha$ can be obtained by Equation 9, when balancing the rotor, $\alpha$ is compensated to the unbalanced phase, so that the adjustment of the dynamic balance is optimal.

The gyro rotor with mandrel is placed on the $\mathrm{V}$-shaped frame of the dynamic balancing machine, and the double calibration method is used to calibrate the dynamic balancing machine to remove the interaction between the front and post correction surfaces when adding the counterweight[5].

The unbalance force and unbalance moment of the rotor are proportional to the square of the rotor speed because of the large initial unbalance of the gyroscope rotor. If the start of the gyro rotor at the rated speed of commissioning, the gyroscope mechanical structure has a greater damage. In order to protect the gyro rotor, the method of grading debugging can be used. The rotor unbalance is reduced to an acceptable range under low speed, and then two times balance is carried out in the gyro's rated rotational speed. Table 1 shows the dynamic balance debugging results of gyro rotor at 3000rpm and table 2 shows the dynamic balance debugging results at $6000 \mathrm{rpm}$.

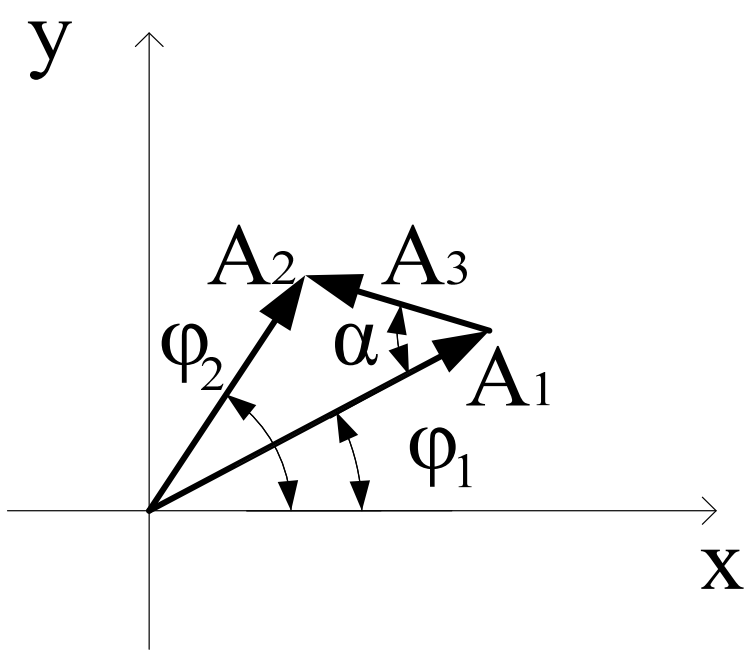

Fig. 4. The diagram of unbalance amount amplitude and phase.

\section{Adjustment of gyro drift amount}

The center of gravity of gyro rotor after dynamic balance adjustment coincides with the rotating axis of rotor, but it does not guarantee that the center of gravity of the rotor coincides with that of the universal joint. According to the law of universal gravitation, this deviation exerts a constant moment to the gyro rotor, which leads to the drift motion of the gyro rotor, and the drift direction of the rotor can be determined according to the right-hand rule. By adjusting the quality and position of the balance screws at the front correction surface center of the rotor as shown in figure 1, the center of gravity of the gyroscope rotor and the rotating center of the universal joint are coincident, and the rotor drift is reduced. Because the balance screw is located at the axis of the rotor, the dynamic unbalance of the gyro rotor will not be affected.

The gyro rotor drift can be initially adjusted by visual inspection. When the drift is smaller, the gyro rotor drift can be calculated by adding an infrared simulation target in front of the gyro, canceling the gyro rotor's electric lock, and the change size and time of the detector imaging. The position and quality of the balance screws are adjusted repeatedly until the rotor drift is in accordance with the technical requirements. 
Table 1. Rotor dynamic unbalance measurement at 3000rpm.

\begin{tabular}{|c|c|c|c|c|}
\hline & \multicolumn{2}{|c|}{ Front correction surface } & \multicolumn{2}{c|}{ Post correction surface } \\
\cline { 2 - 5 } & $\begin{array}{c}\text { Amplitude } \\
(\mathrm{mg})\end{array}$ & Phase $\left(^{\circ}\right)$ & $\begin{array}{c}\text { Amplitude } \\
(\mathrm{mg})\end{array}$ & Phase $\left(^{\circ}\right)$ \\
\hline Initial state & 238 & 43 & 219 & 145 \\
\hline First correction & 132 & 86 & 117 & 181 \\
\hline $\begin{array}{c}\text { Second } \\
\text { correction }\end{array}$ & 44.2 & 157 & 36.7 & 266 \\
\hline $\begin{array}{c}\text { Third } \\
\text { correction }\end{array}$ & 21.4 & 227 & 16.5 & 304 \\
\hline
\end{tabular}

Table 2. Rotor dynamic unbalance measurement at 6000rpm.

\begin{tabular}{|c|c|c|c|c|}
\hline \multirow{2}{*}{} & \multicolumn{2}{|c|}{ Front correction surface } & \multicolumn{2}{c|}{ Post correction surface } \\
\cline { 2 - 5 } & $\begin{array}{c}\text { Amplitude } \\
(\mathrm{mg})\end{array}$ & Phase ( $\left.{ }^{\circ}\right)$ & $\begin{array}{c}\text { Amplitude } \\
(\mathrm{mg})\end{array}$ & Phase $\left(^{\circ}\right)$ \\
\hline Initial state & 25.3 & 295 & 38.3 & 335 \\
\hline First correction & 25.9 & 280 & 26.5 & 349 \\
\hline $\begin{array}{c}\text { Second } \\
\text { correction }\end{array}$ & 21.8 & 316 & 14.1 & 81 \\
\hline Third correction & 8.6 & 322 & 10.7 & 79 \\
\hline
\end{tabular}

\section{Conclusion}

By using the mandrel to eliminate the two deflection degrees of the universal joint, the dynamic balancing machine can directly measure the unbalance force and unbalance moment of the single braced frame gyroscope rotor, and simplify the balancing process of the gyroscope rotor.

\section{References}

1. Y.B. Zhou: A Course in Theoretical Mechanics (HE Press, China, 1985)

2. A.C. Wang, Z.H. Yu, JMAG, 20(2000)

3. N.A. Ye, R.S. Yu: Dynamic Balancing Principle and Dynamic Balancing Machine (HITH Press, China 1985)

4. Y.J. Lu: Gyro and Inertial Navigation Principle (Science Press, China 1964)

5. J.G. Lee, C.G. Park, H.W. Park, IEEE TAES, 28(1992) 